\title{
Optical and structural properties of InN grown by HPCVD
}

\author{
M. Buegler ${ }^{1}$, M. Alevli ${ }^{1}$, R. Atalay ${ }^{1}$, G. Durkaya ${ }^{1}$, I. Senevirathna ${ }^{1}$, M. Jamil ${ }^{2}$, I. Ferguson ${ }^{2}$ and \\ N. Dietz ${ }^{1}$ \\ ${ }^{1}$ Physics \& Astronomy Department, Georgia State University, Atlanta, GA, 30303 \\ ${ }^{2}$ School of ECE, Georgia Institute of Technology, Atlanta, GA, 30332
}

The optical and structural properties of InN layers grown by 'High Pressure Chemical Vapor Deposition' (HPCVD) using a pulsed precursor approach have been studied. The study focuses on the effect of ammonia precursor exposure time and magnitude on the InN layer quality. The samples have been analyzed by X-ray diffraction, Raman scattering, infra red reflectance spectroscopy and photoluminescence spectroscopy. Raman measurements and X-ray diffraction showed the grown layers to be single phase $\mathrm{InN}$ of high crystalline quality. The $\mathrm{E}_{2}$ (high) Raman mode showed FWHM's as small as $9.2 \mathrm{~cm}^{-1}$. The FWHM's of the InN(0002) X-ray Bragg reflex in the $2 \Theta-\Omega$-scans were around 350 arcsec, with rocking curve values as low as 1152 arcsec Photoluminescence features have been observed down to $0.7 \mathrm{eV}$, where the low energy cutoff might be due to the detector limitation. The analysis of the IR reflectance spectra shows that the free carrier concentrations are as low as $3.3 \cdot 10^{18} \mathrm{~cm}^{-3}$ for $\mathrm{InN}$ layers grown on sapphire substrates.

\section{Introduction}

Research in growth and analysis of group-III nitride semiconductor alloys are driven due to their huge potential for optoelectronics, high-speed electronics, multi-functional sensors, as well as unique detectors that covers the wavelength regime from UV down to the far IR. Especially, the integration of indium-rich group III-nitride alloys - even though their physical and optical properties are not well understood - is of crucial importance in order to assess their prospects within the group III-nitride material system.

Even though, significant progress has been made in recent years in the growth and understanding for the binary group III-nitride systems ( $\mathrm{GaN}, \mathrm{AlN}$, and $\mathrm{InN})$, the formation of ternary/ quaternary alloys and the studies of their properties is a challenge due to two factors:

a) the alloying between two binaries is always related with a change in lattice constants, inducing a strain effect - which is for instance negligible in the GaAlAs system

b) the ternary/ quaternary alloys have to bridge large differences in partial pressures between the binaries, which leads is vastly different process growth conditions - specially in largely different growth temperatures for low-temperature CVD processing.

The latter factor is one of the main challenges in the growth of $\mathrm{InN}$. Due to the low decomposition temperature of $\mathrm{InN}$, the growth temperature in low-pressure $\mathrm{CVD}$ is $600^{\circ} \mathrm{C}$ or below ${ }^{1}[1]$. The cracking efficiency of ammonia $\left(\mathrm{NH}_{3}\right)$ in this temperature regime is extremely small, requiring large group V-III precursor ratio[2]. 
The difference in the growth temperature of $\mathrm{GaN}$ and $\mathrm{InN}$ results in the decomposition in indium-rich alloys when grown at or near optimal GaN growth conditions. The question that arrises is whether there exist process conditions under which two alloys with vastly different partial pressures can be stabilized at the same growth temperatures?

The approach chosen in our group is based on the studies of 'thermal stability of indium nitride at elevated temperatures and nitrogen pressure' by MacChesney et al.[3], who suggested already 1970 from thermodynamic equilibrium calculation that a growth surface of group III-nitrides can be stabilized with nitrogen overpressure. The analysis is summarized in Fig.1 and show that the growth temperature can be significantly increased if the reactor pressure can be utilized as an additional process parameter. Even though the pressure dependency the chemical reactions is well established in many chemical processes, its utilization within the semiconductor thin film process technology is still lacking, since it was not required for Silicon technology was well as for the group III- arsenide or group III- phosphide systems.

The work at GSU explores the pressure dependent growth parameters for $\mathrm{InN}$ and indium-rich $\mathrm{In}_{1-\mathrm{x}} \mathrm{Ga}_{\mathrm{x}} \mathrm{N}$ alloys, using a custom-built high-pressure flow-channel reactor with precise temporal controlled precursor injections capabilities. The precursor fluxes are embedded in the main carrier gas stream, maintaining constant flow and pressure at all times.

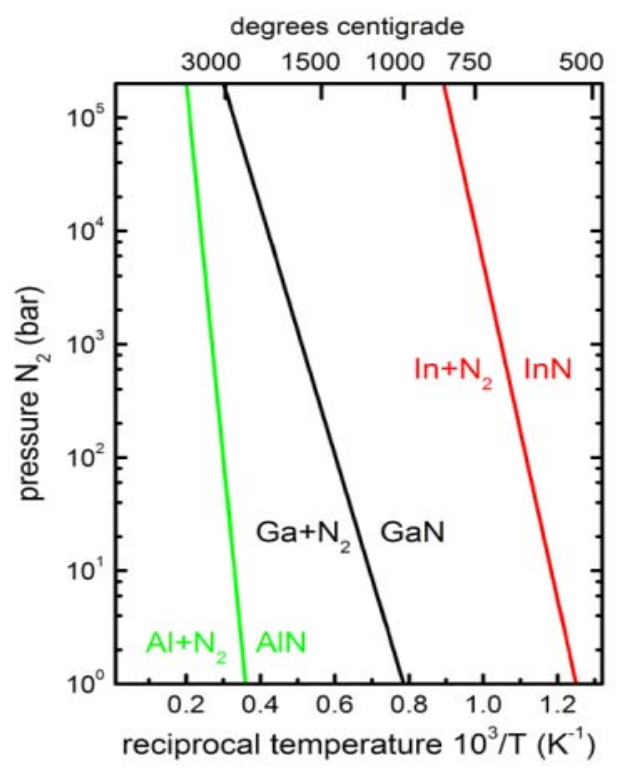

\section{Figure 1:}

Diagram of thermal stability of III-nitrides under elevated pressure[3]

\section{Experimental conditions}

All InN layers analyzed and discussed here were grown by high-pressure MOCVD, utilizing a reactor pressure of 15 bar. The precursors for indium and nitrogen were Trimethylindium (TMI) and ammonia $\left(\mathrm{NH}_{3}\right)$, respectively. As depicted in Fig. 2, the precursors are deployed temporally controlled with an overall injection cycle of $6 \mathrm{sec}$. For the growth series analyzed here, the TMI injection time was $0.8 \mathrm{sec}$ and separated $1.4 \mathrm{sec}$ from the ammonia injection pulse, the pulse length of which was varied from $1.5 \mathrm{sec}$ to $2.5 \mathrm{sec}$. The magnitude of the ammonia pulse was 
adjusted to maintain a constant group V/III precursor ratio of 2110 . The growth temperature was also kept constant at $865^{\circ} \mathrm{C}$.

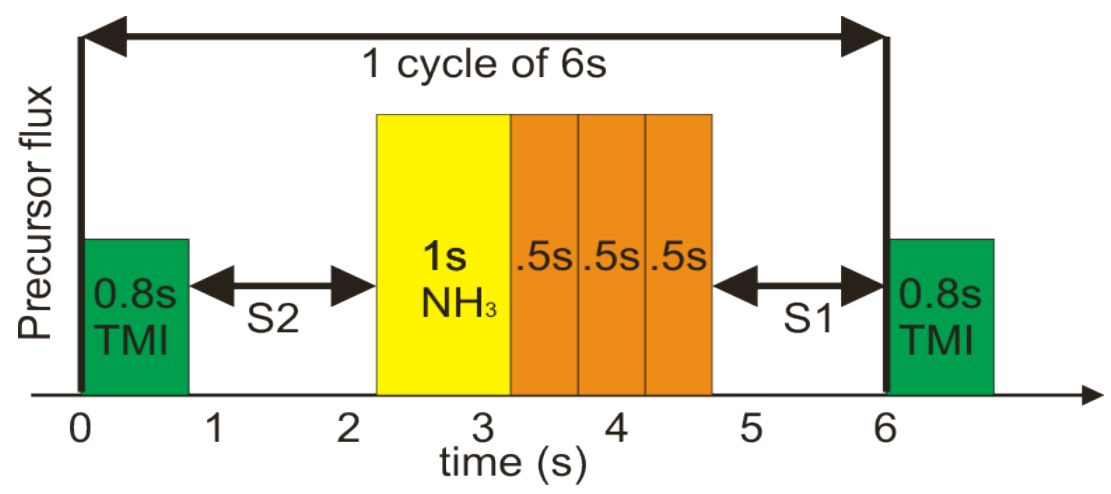

Figure 2:

Scheme of pulsed

precursor injection

After establishing the reactor pressure of $15 \mathrm{bar}$, the substrate was heated up to a temperature of $800^{\circ} \mathrm{C}$ and nitridized for $2 \mathrm{~min}$, an initial nucleation buffer with a low V/III-ratio of around 500 was deposited, using 10 precursor injection cycles $(1 \mathrm{~min})$. The nucleation buffer was then annealed for $9 \mathrm{~min}$ and at the same time the temperature was increased to the InN growth temperature of $865^{\circ} \mathrm{C}$. As the growth is reached, the growth is established with a V/III precursor ratio of 2100. After $180 \mathrm{~min}$ growth time, the TMI flux is stopped and the growth surface remained exposed to pulsed ammonia exposure for additional 15 cycles, before the temperature of the growth surface is lowered. The samples are cooled down while maintaining the reactor pressure and flow of the nitrogen carrier gas.

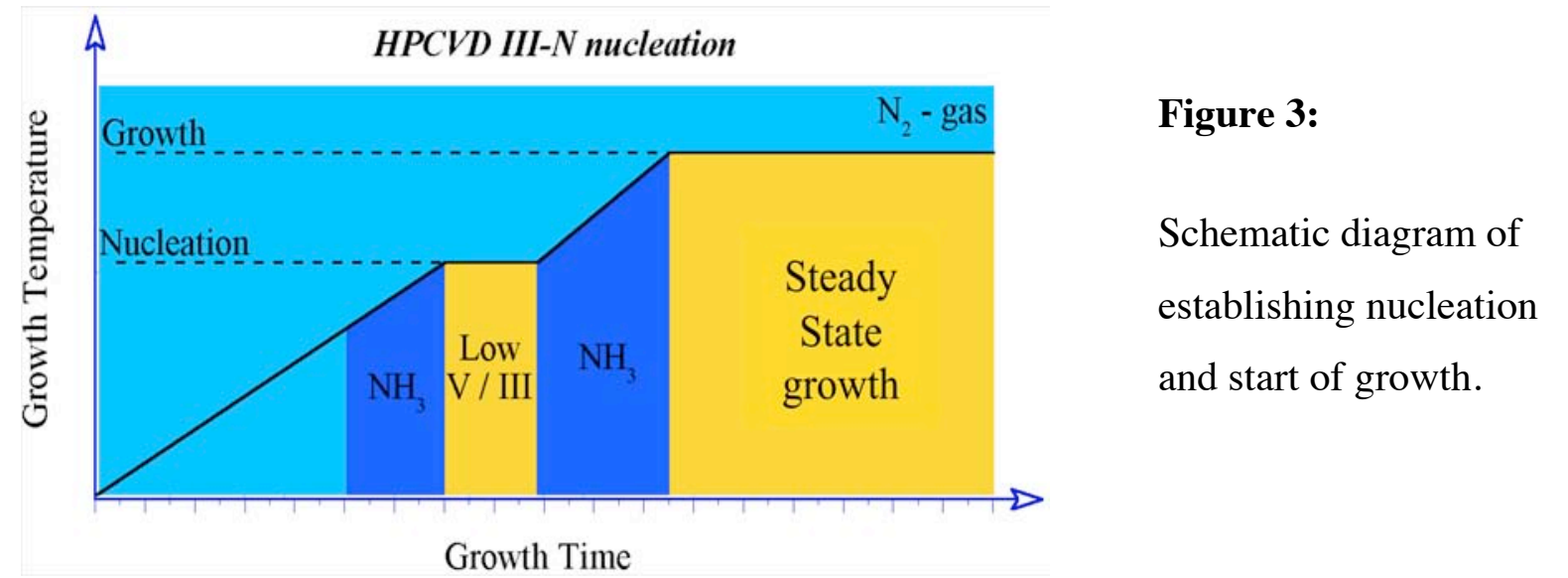

\section{Structural analysis}

XRD $2 \Theta-\Omega$-scans and Rocking curves performed on the InN layers clearly indicate the growth of single-phase layers of high crystalline quality. The $\operatorname{InN}(0002)$ Bragg reflexes in the $2 \Theta-\Omega$ scans have full-width-half-maxima (FWHM) values in the order of 355 arcsec. The Rocking curves show single Bragg reflexes with FWHM's in the range of 1152 arcsec. Figure 4 shows the effect of varying the ammonia exposure length in each injection cycle on the crystalline quality as analyzed by $2 \Theta-\Omega$ XRD scans as well as Rocking curve analysis. 


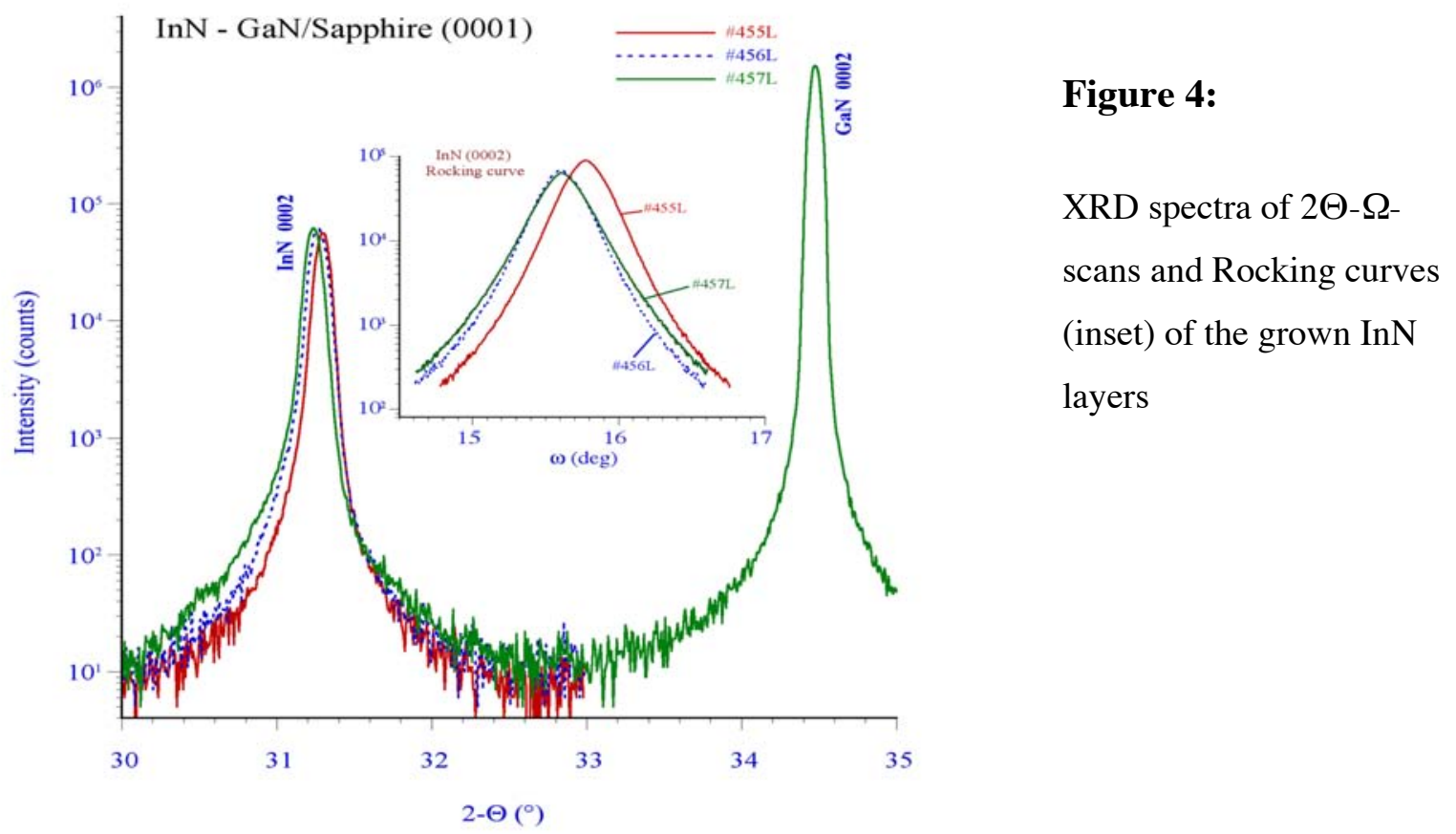

The analysis of the FMHM's values in the InN(0002) Bragg reflexes with the ammonia exposure length is summarized in Fig. 5. The FWHM's values for the Bragg reflexes of the $2 \Theta$ - $\Omega$-scans and the Rocking curves reduce as the ammonia exposure time increases to about $2 \mathrm{sec}$. A further increase in the ammonia exposure time of the growth surface leads to an significant increase of the FWHM in the $\operatorname{InN}(0002)$ Bragg peak in the $2 \Theta-\Omega$-scan, while the FWHM in the Rocking curve Bragg reflex remains almost unchanged, the FWHM of the $2 \Theta-\Omega$-scan increases by $16.6 \%$.

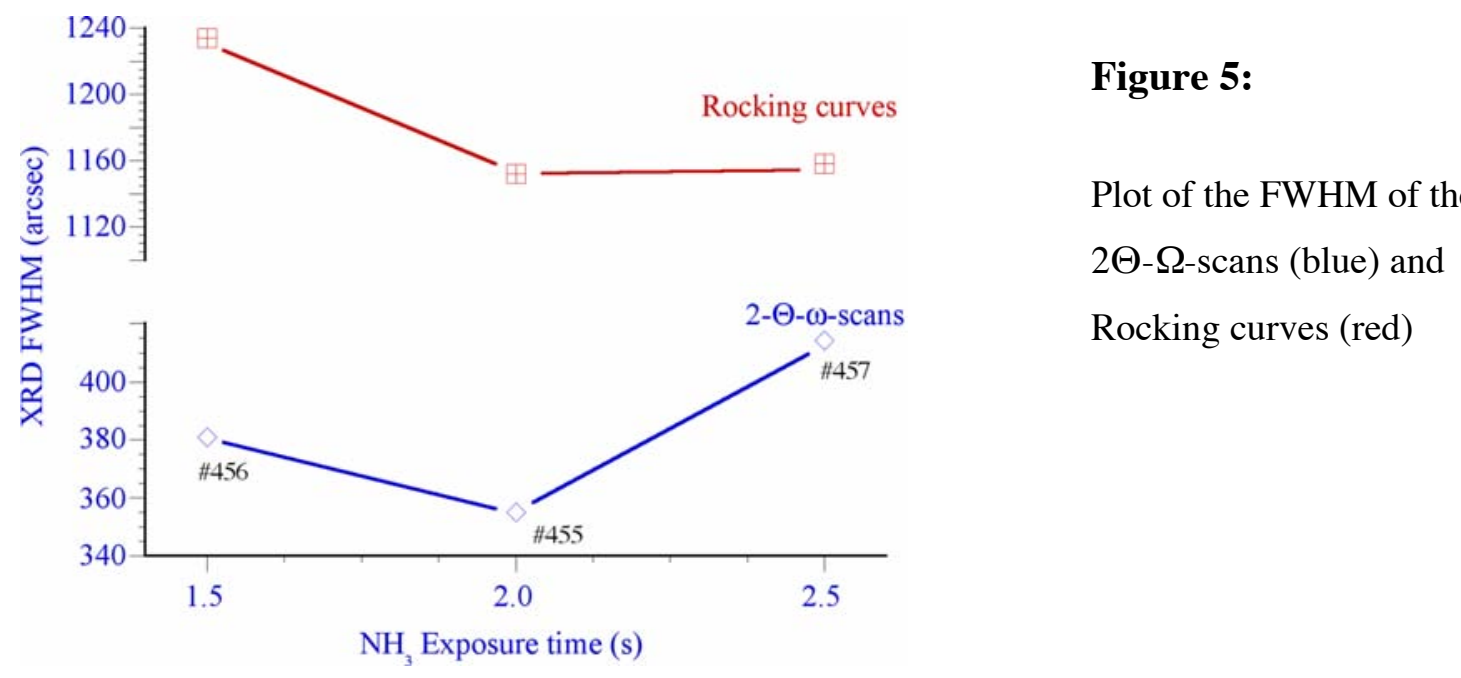

The behavior depicted in Fig. 5 shows that a short exposure time increases the incorporation of point defects as well as it decreases the overall crystalline quality, whereas an increase of the exposure time beyond 2 seconds strongly decreases the crystalline quality but does not much increase the amount of point defects and dislocations[4]. 


\section{Optical properties}

IR reflectance spectroscopy is used to analyze the free carrier concentrations and mobility in the InN layers. Figure 6 shows the experimental measured as well as the resulting parameters of the simulation of the spectra for an InN layer deposited directly on sapphire. The simulation - using a multi layer stack model - estimates a free carrier concentration of $3.3 \cdot 10^{18} \mathrm{~cm}^{-3}$. IR reflectance spectra for layers grown on GaN/sapphire templates revealed free carrier concentrations as high as $2 \cdot 10^{19} \mathrm{~cm}^{-3}$ for similar growth conditions.

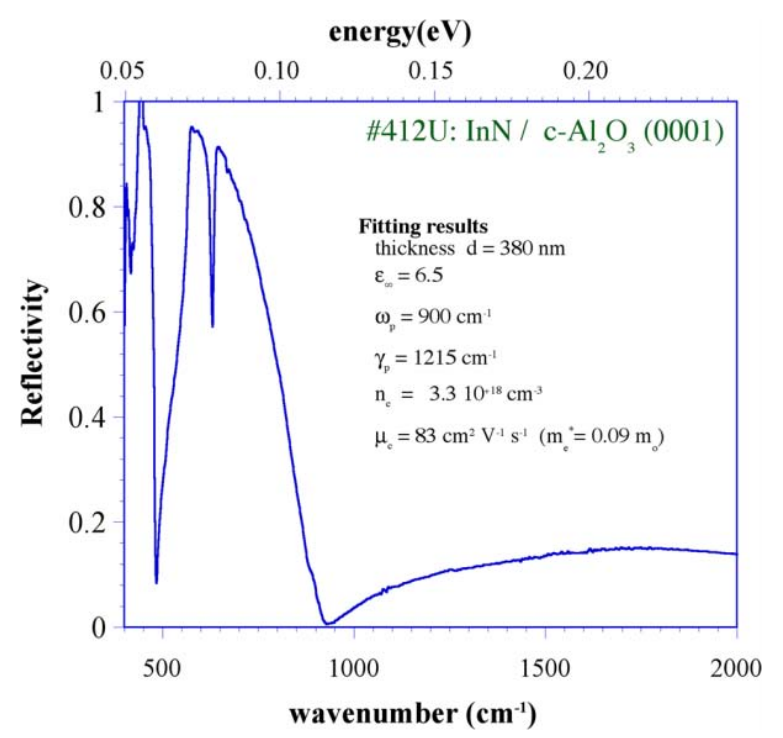

\section{Figure 6:}

IR reflectance spectra of an InN layer grown directly on sapphire

The photoluminescence (PL) spectrum of a sample grown under similar conditions is shown in Fig. 7. It shows a Luminescence at $0.77 \mathrm{eV}$ with a FWHM of $0.02 \mathrm{eV}$. Since the germanium detector used has a sharp fall off at $0.75 \mathrm{eV}$, we cannot rule out further features at lower energy. We attribute this luminescence due to good agreement with absorption measurements, which showed an absorption edge below $0.8 \mathrm{eV}$ to band edge transitions.

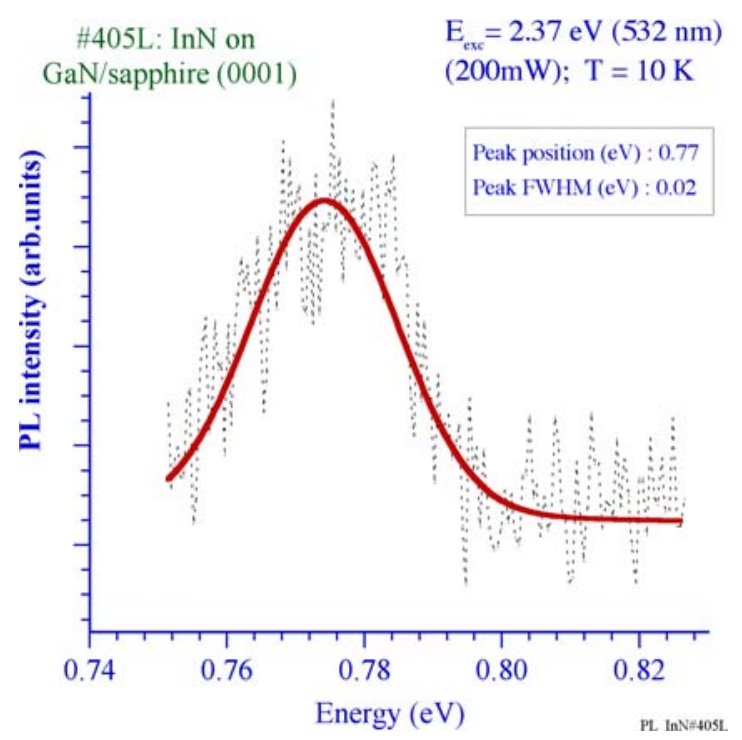

\section{Figure 7:}

PL spectra of an InN layer grown on a $\mathrm{GaN}$ on sapphire template (fall-off below $0.75 \mathrm{eV}$ due to $\mathrm{Ge}$ detector limitations) 
Further investigation on the causes for the observed lowered free carrier concentration for layers grown directly on sapphire are ongoing.

\section{Conclusion}

We showed that the utilization of high-pressure CVD enables the successful growth of high crystalline quality layers of $\mathrm{InN}$ on sapphire and $\mathrm{GaN} /$ sapphire templates. At a reactor pressure of 15 bar, the growth temperature for $\mathrm{InN}$ can be raised to about $850^{\circ} \mathrm{C}$, which will allow the growth of indium-rich alloys embedded into wide band gap materials like GaN. For an explored $6 \mathrm{sec}$ precursor injection cycle during the growth of InN, optimum crystalline quality of the layer was found for an ammonia exposure time in the range of 2 seconds for a group V/III precursor ratio of 2100. Photoluminescence studies show a PL structure in the range of $0.7 \mathrm{eV}$ for layers with free carrier concentrations in the upper $10^{18} \mathrm{~cm}^{-3}$ range, as determined by IR reflectance. Further investigations have to be carried out for different group V/III precursor ratio to establish the optimum surface chemistry, leading to better crystalline quality and reduce free carrier concentration in the layers. A further puzzle is the observed large difference in free carrier concentrations in layers grown on $\mathrm{GaN} / \mathrm{sapphire}$ templates compared to InN layers grown directly on sapphire.

\section{Acknowledgement}

This work has been supported by AFOSR under the award \#FA9550-07-1-03450 and by GSU GRP. The authors also acknowledge the support of Dr.'s Hoffmann (TUB) and Sitar (NCSU) research groups.

\section{$\underline{\text { References }}$}

1. Bhuiyan, A.G., A. Hashimoto, and A. Yamamoto, Indium nitride (InN): A review on growth, characterization, and properties. Journal of Applied Physics, 94(5), p. 27792808 (2003)

2. Dietz, N., M. Strassburg, and V. Woods, Real-time Optical Monitoring of Ammonia Decomposition Kinetics in InN Vapor Phase Epitaxy at Elevated Pressures. Journal of Vacuum Science \& Technology A: Vacuum, Surfaces, and Films, 23(4): p. 1221-1227 (2005)

3. MacChesney, J., P.M. Bridenbaugh, and P.B. O'Connor, Thermal stability on Indium Nitride at elevated temperatures and nitrogen pressures. Mater. Res. Bull., 5: p. 783 (1970)

4. Chierchia, R., et al., Microstructure of heteroepitaxial GaN revealed by x-ray diffraction. Journal of Applied Physics, 93(11): p. 8918-8925 (2003) 